majority of patients were symptomatic (92\%): jaundice (68\%), abdominal pain (35\%), fever (17\%) and rash (15\%). Hepatic encephalopathy was present in $32 \%$. Laboratory patterns of DILI were: hepatocellular ( $\mathrm{R}$ value $>5) 54 \%$, cholestatic $(\mathrm{R}<2)$ $28 \%$ and mixed $(\mathrm{R}=2-5)$ 19\%. Hy's law was met in $48 \%$ while $26 \%$ had ALF (encephalopathy + INR $>1.5$ ). The median admission MELD score was 21. 35\% of patients received corticosteroids, and $15 \%$ received ursodeoxycholic acid. ICU admission and haemodialysis occurred in 35\% and $11 \%$, respectively. During the study period, there were 12 deaths and 12 LT. The 90-day LT-free survival was 71\%. Univariate predictors for LT or mortality at 90 days were: jaundice (HR 9.77, $\mathrm{P}=0.027)$, encephalopathy (HR 2.70, $\mathrm{P}=0.036)$, hepatocellular pattern (HR 2.85, $\mathrm{P}=0.047)$, fulfilling Hy's Law (HR 2.71, $\mathrm{P}=0.046$ ) and MELD (HR 1.14, $\mathrm{P}<0.001)$. On multivariable analysis, only MELD remained predictive of worse 90-day LT-free survival (HR 1.14 per point increase, $\mathrm{P}<0.001)$.

Conclusions At this LT centre, 30\% of patients hospitalised for non-paracetamol DILI experienced death or LT at 90 days. The proportion of cases due to non-prescription drugs increased over time. MELD score predicted for adverse outcomes.

\section{IDDF2020-ABS-0169 DEVELOPMENT OF VIROLOGICAL BREAKTHROUGH IN TREATMENT NAÏVE HEPATITIS B PATIENT RECEIVING TENOFOVIR: A CASE REPORT}

Ignatius Bima Prasetya*, Nata Pratama Hardjo Lugito, Andree Kurniawan. Department of Internal Medicine, Faculty of Medicine Pelita Harapan University, Indonesia

\subsection{6/gutjnl-2020-IDDF.159}

Background Tenofovir disoproxil fumarate (TDF) is a nucleotide analogue that is widely used to treat chronic hepatitis B infection. This treatment is currently considered to be effective in achieving good virological, serological, and biochemical response with a high barrier of resistance. We reported a case of a virological breakthrough in a patient with chronic hepatitis $\mathrm{B}$ and cirrhosis receiving TDF.

Methods We presented a case of a 48-year-old male who had been treated with TDF for the last 10 months.

Results The patient was diagnosed with decompensated cirrhosis with variceal bleeding and was tested positive for hepatitis B. his initial viral load prior to treatment was $4.38 \times 10^{4} \mathrm{IU} /$ $\mathrm{mL}$. Four months after the initiation of the antivirus, his serum HBV DNA level was undetectable, and there were improvements in biochemicals parameters. However, the serum HBV DNA level rebounded to $1.28 \times 10^{3} \mathrm{IU} / \mathrm{mL}$ at 10 months after treatment. The patient was compliant with the treatment program, was monitored regularly, and took his medication every day. No prior history of other antiviral agents was noted, and he didn't have any specific comorbidity. $\mathrm{He}$ is in otherwise stable clinical condition. We are planning on switching his treatment to entecavir.

TDF is one of the only 2 antivirus agents (along with entecavir) that was thought to have a high barrier of resistance. A longitudinal study of TDF therapy demonstrated no resistance development throughout 8 years of treatment, although several case reports have identified resistance cases. Several studies had pointed out possible mutations' points for TDF resistance, including A181T/V, A194T, M204V/I, Y9H, L91I, S106C,
S106G, T118C, T118G, Q267L, I269L, A317S, K333Q, and $\mathrm{N} 337 \mathrm{H}$. Switching treatment to entecavir seemed to show good results in previous reports.

Conclusions The virological breakthrough might still occur in patients receiving TDF. Further evaluation of such resistance mechanism was needed.

\section{IDDF2020-ABS-0171 ACUTE LIVER FAILURE: OUTCOME AND PROGNOSTIC PREDICTORS}

${ }^{1}$ Jalpa Devi* ${ }^{2}$ Mohsin Ali, ${ }^{1}$ Amerta Bai, ${ }^{2}$ Adil Hassan, ${ }^{2}$ Saadat Ali, ${ }^{2}$ Muhammad Sadik. ${ }^{1}$ Liaquat University of Medical and Health Sciences, Pakistan; ${ }^{2}$ Asian Institute of Medical Sciences, Pakistan

\subsection{6/gutjnl-2020-IDDF.160}

Background Acute liver failure (ALF) is defined as a rapid hepatic dysfunction and encephalopathy in the absence of preexisting liver disease. Globally, viral hepatitis is responsible for the majority of cases of ALF. This study aimed to determine the etiology, outcome, and predictive factors for in-hospital mortality in ALF patients.

Methods A descriptive study was conducted at the GastroHepatology Department of Asian Institute of Medical Sciences, Hyderabad from May 2018 to September 2019. A total of 31 patients were included in the study and evaluated for etiology, prognostic factors, and outcome during the hospital stay. International Normalized Ratio (INR), sepsis (2 SIRS + confirmed or suspected infection), prognostic scores \{King College Criteria (KCC), and Model End-Stage Liver Disease (MELD) $\}$ and other prognostic factors were compared.

Results Thirty-one patients with a mean age of 22 years, 21 (67.7\%) were males. Most common etiology was indeterminate $21(67.7 \%)$ while $5(16.15 \%)$ had Hepatitis B and 5 $(16.15 \%)$ had Hepatitis E. The in-hospital mortality was 19 $(61.3 \%)$, out of which $14(73.3 \%)$ were males and 12 (38.7\%) recovered spontaneously. INR $>5.00$ (Mean= 3.12 and 4.02 in both groups respectively, $p=0.02$ ), MELD score $>32($ Mean $=29.58$ and 33.31 in both groups respectively, $p=0.049$ ), KCC 2 or more out of 5 (Mean $=0.83$ and 1.31 in both groups respectively, $p=0.068)$, and sepsis $(p=0.008)$ were independently associated with in-hospital mortality.

Conclusions The in-hospital mortality of ALF was significantly high with raised INR, MELD (>32), KCC (2/5), and sepsis. Hence, they are poor prognostic factors.

\section{IDDF2020-ABS-0172 THIRTY-DAY READMISSION AFTER ESOPHAGEAL VARICEAL HEMORRHAGE AND ITS IMPACT ON OUTCOMES IN THE TERTIARY CARE HOSPITAL}

${ }^{1} J a l p a$ Devi*, ${ }^{1}$ Amerta Bai, ${ }^{1}$ Rabia Farooque, ${ }^{1}$ Riaz Hussain Awan, ${ }^{2}$ Saadat Ali, ${ }^{2}$ Muhammad Sadik. 'Liaquat University Of Medical and Health Sciences, Pakistan; ${ }^{2}$ Asian Institute of Medical Sciences, Pakistan

\subsection{6/gutjnl-2020-IDDF. 161}

Background Esophageal variceal hemorrhage $(\mathrm{EVH})$ is a potentially fatal Gastro-intestinal emergency. The aim of this study was to evaluate the in-hospital mortality rate, 30-day readmission rate, and its impact on mortality and morbidity in EVH patients.

Methods A descriptive study (prospective) was conducted at the Gastro-hepatology department of AIMS Hyderabad from 